\title{
Functional Analysis and Library Science
}

Functional analysis is a tool with significant positive implications for library research because of: (1) its facility for investigating the social system of which the library is an integral part; and (2) its direction towards heuristic considerations and metaphysical theory long neglected in library science. The sociological approach rather than the mechanistic or mathematical approach to functionalism should be adopted by library researchers because of its ability to develop functional relationships rather than deterministic, causal relationships.

$\mathrm{F}_{\mathrm{c}}$ methodology that has received consid erable attention from the disciplines in the social sciences. Because the library is an important element in the social system and is initially concerned with human educational behavior, it is logically consistent that librarians consider adopting a research method that has been highly effective in other socially scientific areas.

For the librarian, the functional approach to analysis has particular advantages. These advantages stem from the nature of phenomena in library science and from the nature of the functional method.

What is significant about the functional method is that it is an analytic process which attempts to establish dependencies between phenomena which are considered to be essential and interrelated parts of an integral whole. Consequently, the functional approach to investigation is highly adaptable to elements in the social system. Social scientist Claude Levy-Strauss, for example, stresses that "all aspects of social life, all institutions, whether social, politico-legal, economic, technological, re-

Mr. Christ is Director of the Library at Rockhurst College in Kansas City. ligious, or aesthetic, form a related whole." 1 The library is contained within that related whole to which Levy-Strauss refers. And, because of the widespread nature of the library's educational, economic, social, and political activities, understanding the complexity of these relationships requires an analytic system which will maximize the interrelatedness of these varied social relationships.

The premise of the functionalist position is that no human custom, social institution, or set of behaviors exist in a vacuum, that there must always be an interplay between the component elements which comprise the social system, and that a continuing interdependence between them is created on many different levels. ${ }^{2}$

Functionalism is not a particularly elaborate or complex conceptual framework within which to work. Philosophereconomist I. C. Jarvie defines functionalism as an anytic tool very succinctly. "As a rough first approximation," he said, "we can say it is a method of ex-

\footnotetext{
${ }^{1}$ Claude Levy-Strauss, Structural Anthropology, tr. by C. Jacobson and B. C. Schoepf (New York: Basic Books, 1963), p. 277.

${ }^{2}$ Robert F. Spencer, "The Nature and Value of Functionalism in Anthropology," in The American Academy of Political and Social Science, Functionalism in the Social Sciences (Philadelphia: AAPS, 1965), p. 13 .
} 
plaining social events and institutions by specifying the functions they perform."

The overall development of the functional approach to investigation in science has taken two approaches. Jarvie explained,

At this stage we can split functionalism in two: the theory that every action or institution has a function or functions, and the theory that societies are well integrated, well adjusted, and seamless. The first assumption depends upon the second; it is because societies are seamless that all actions and institutions have a function. The first theory asserts the existence of functions: (i.e.) "There exists a function for all events and institutions." The second theory makes a factual assertion about the character of existent societies: "Present societies contain no nonfunctional elements." The first is useful but unfalsifiable; the second is restrictive and false. Insofar as functionalism assumes a well-integrated, efficiently adjusted, and seamless society, it is taking on the character of a metaphysical theory, that is, a theory that cannot be shown to be false. ${ }^{4}$

Jarvie's statement pertains to the philosophy of the functional approach to scientific investigation. It follows from a validity standpoint that functionalism and the functional approach are limited in that they cannot be verified; that is, the results of functional analysis cannot be tested at any mathematical degree of confidence, but only in the general terms of logical consistency. Such a limitation is particularly relevant, however, only if the results of the investigation require, or are thought to require statistical verification. Such verification, however, is generally associated with quantitative measurement. The functional approach to the library as a social institution within a social system is highly qualitative or attributive. Thus, while a functional analysis of the library does take on the character of a metaphysical

s Ibid., I. C. Jarvie, "Limits to Functionalism and Alternatives to It in Anthropology," p. 19.

Ibid., p. 27. theory this is not particularly debilitating. The verification of the results of such an investigation will pertain more to the logical consistency of the results than to their being verified at a statistical level of confidence.

The functional approach to investigation is also relevant for the library from another point of view. This pertains to the trends which the application of functionalism have taken. Used in a mathematical or mechanistic sense, functional analysis has taken a rigid, deterministic bent. Mathematical functionalism expresses the relationships between two elements, two phenomena, or two or more variables stating a condition or conditions of the relation or relationships. For example, the mathematical approach to functional analysis might state that for every value of one phenomenon there are one or more determinate values of the other phenomenon. This type of functionalism, therefore, includes the notion of a causal relationship between independent and dependent variables. That is, the second phenomenon, the dependent variable, is said to be a function of the first phenomenon or independent variable. If society were considered as the independent variable, the library, education, government, hospitals, all social phenomena could be treated as dependent variables. If the library were considered as the independent variable in analysis, then social institutions as well as the patron, book materials, etc., could be considered as dependent variables of the library as a social entity.

The use of the mathematical or mechanistic approach to functionalism, however, has less applicability in library science than the second or sociological approach to functional analysis. The nature of the phenomena in library science seems to be less mutually exclusive than that of mathematics or the physical sciences. Therefore, phenomena pertaining 
to the library are also dynamic rather than highly static, or rigid. Sherman Roy Krupp discussed the applicability of the mechanistic approach to functional analysis in economics. He said:

Functional theory focuses on the unity and directedness of a total system, while mechanistic theory tends to concentrate on the precise determination of the relationship between parts of a system. . . . In mechanical theories the parts are assumed to be independent entities which are combined to special rules to yield aggregates. These aggregates obey the same general laws that apply to the parts. ${ }^{5}$

To discuss relationships between the library and other elements in the social sphere in terms of general mechanical laws is not valid. Such an approach requires statistical validation because of the implication of certitude that is involved. Thus, such an approach is not applicable to library science and, therefore, not an effective manner to undertake scientific investigation.

An approach more suited to library analysis is what is commonly called the sociological functional theory. Krupp referred to this method of functional analysis as a "theory (which) assumes a system to have a basic organizing principle of goals and self-regulating mechanisms."6

The sociological approach to functional analysis has a greater degree of relevance for library research than the mechanistic approach because the method is more closely related to the type of phenomena and social system which comprises the library as an institution. The mechanistic approach, because of its assumed rigidity of the phenomena and relationships involved naturally leads to a cause-effect, deductive-inductive approach to research and scientific analysis. This research method, often associated or equated with the historical

"Ibid., Sherman Roy Krupp, "Equilibrium Theory in Economics and in Functional Analysis as Types of Explanation," p. 65, p. 70.

Ibid. or comparative methods, has typified most library research in the past. Anthropologist Robert F. Spencer points to the limitations of the deductive-inductive method for evaluating social phenomena and to the advantages of the functional approach when dealing with social reality. Spencer stated:

In general terms, however, a problem of causality is at base a problem in history, one founded in a diachronic rather than a synchronic method. The methodology of functionalism sees sociocultural systems as ends, thereby ruling out the cross-cultural comparisons which have been so essential to historical formulations. . . . Comparison of an institution between several societies, as in the case of the couvade above, is clearly different from analyzing it in relation to its place in a single social entity. In the latter case the issue is one of mode rather than of cause. ${ }^{7}$

The close correlation between previous library research which has been largely historically oriented and the functional method mentioned by Spencer was referred to by Carl M. White. He said:

Writings on library history tend to describe individual libraries or the libraries of a country or period. These accounts can be expected to cover such topics as: the growth of collections, acquisition, classification and cataloging, housing, finance, regulations and personnel. All of this information is good as far as it goes, but too often it leaves the reader to search in vain among sorted facts for some internal order -some mosaic-which will give the several fragments range of meaning. It is of course plain to all that libraries are among the things that are products of human effort, so if some larger configuration of meaning is to be found in their history, it is better to relate them to the whole stream of social evolution than to separate them as it were from human strivings. ${ }^{8}$

Author White's concern is clearly for a functional analysis of the library.

While past library research has pro-

${ }^{7}$ Ibid., Spencer, p. 8.

${ }^{8}$ Carl M. White (ed.) Bases of Modern Librarianship (New York: Macmillan, 1964), p. 1. 
duced a deposit of factual data, it has not developed the kinds of relationships or understanding needed to produce theory for the profession. Many productive authors such as Shera, Butler, and Foskett have consistently pleaded with the profession to develop philosophical concepts and principles in order that theories of relationships and procedures of implementation might be developed. The underlying assumptions of such a point of view are that before a philosophy can be defined working theories must be uncovered and examined for their applicability to the phenomena of the discipline and the type of system which constitutes the library. Barbara G. Petrof made a plea similar to those mentioned above. "Librarians," she stated, "have come to recognize the value of research to the profession and much collecting of data is being accomplished. . . . There has been little attempt thus far to develop an adequate theoretical framework into which practical research can be hung. Such an examination of the theoretical bases of librarianship will probably have to be made by library educators rather than by practitioners." While this statement is accurate in that there is a drastic need for theory development in library science, the assumption that this will probably occur with library educators may be erroneous. Such development is not taking place in the library schools at this time.

The specific applicability of the sociological approach of functional analysis for library science concerns the treatment of both the phenomena and the system in that approach. Rather than mechanically rigid, this approach allows for interplay and overlap between relationships which define function. This is particularly beneficial in a system which does not possess rigid, determin-

\footnotetext{
"Barbara G. Petrof, "Theory: the X Factor in Librarianship," College \& Research Libraries, XXVI (July $1965), 316$.
}

istic functions, such as the library, or where relationships are known to be definite and constant.

The specific difficulty which library science realizes in maximizing its utilization of the functional method of analysis pertains to the purpose or end of the library. Such a question is basically a philosophical consideration. Library science, however, has not developed a clear, well-defined philosophical base. As a result explanations of the library tend to be forced into a causal orbit where cause-effect relationships exhaust the scope of the research. This deductive-inductive approach, paramount in the history of the discipline, is only one way of deriving explanations. The cause-effect approach to explanation is unilinear where the functional approach is multi-linear. The functional approach, because of its horizontal and vertical thrusts, is a much more advantageous perspective for library theorists because it is involved with the totality of human society rather than just specific, well-defined, rigid aspects of social life. The library, for example, has the potential to be constantly concerned with the life of the individual and with his varied activities. Because the scope of the library's activities covers the whole social spectrum, the potentiality for relationships is enormous. This is not true of other social institutions. The schools, the hospital, the business world, the military, etc., are all concerned with the social person directly only at specific periods or for specific durations of time. From the standpoint of the socialization process the only possible time that the library does not have the potentiality for influence through direct contact with the individual is from birth to about one year of age. Once the socialization process has gained momentum so that the child begins to interact rather than only react to social stimuli, the library may take a continuous part in the child's life. The structure of other 
social institutions, however, prevents influence until specific periods or points in the person's life. It is these relationships to the human person and the end or purpose of the library which need philosophical development and which, being presently underdeveloped, limit functional and operational developments of the library.

Since the library is concerned not only with the records of human activity but with the human person as well, its basic framework is as a social institution. And, since the library exists in social experience with other social institutions, it is necessary, for a proper understanding of the purpose of the library, to consider its relation to society as a whole and to the diverse elements within society which comprise the social framework. The functional approach, being multi-linear, offers more of a chance to achieve such analysis than does the historical, or comparative, approach. These linear approaches to investigation do offer advantages in subanalysis in that they offer the researcher dealing with the library rigid parameters within which to work. Thus, understanding the internal functions of the library as a closed system could be achieved by both a functional and deductive-inductive methodology.

Anthropologist Robert F. Spencer takes an optimistic approach to functional analysis. "The functional approach is at base simple," he said, "it seeks to do no more than assay the place of a particular element of culture or societal institution in relation to other elements."10

The casual tone in Spencer's remarks

${ }^{10} \mathrm{Op}$. cit., Spencer, p. 1. should not suggest that functional analysis is to be viewed as the panacea of research. Quite the contrary. Functional analysis is only one of many approaches to scientific investigation, all of which should be evaluated for their applicability to library science. Functionalism does have disadvantages which should be noted.

I. C. Jarvie pointed out one general disadvantage to functionalism which happens, at this stage in the history and development of library science, to have positive significance for the field. Jarvie said, "I shall argue, its lack of explanatory power, its unsatisfactoriness as explanation, and the constricting effect of its assumptions about the nature and working of social systems. Its merits are mainly heuristic. ${ }^{11}$

Jarvie's complaints echo statements made elsewhere to the effect that the main fault of the functional method as an analytic tool is its lack of verifiability and its tendency to be nothing more than metaphysical theory. As Jarvie said, its merits are mainly heuristic. But this is the point. What is needed in library science at this point in time and at this stage of its professional development is metaphysical theory. Functional analysis leads directly towards heuristic considerations. Hopefully, out of functionalistic considerations to be developed in the future by library theorists and researchers will come the functional theories and principles which will constitute the basis for a philosophy of library science which has long been called for by leading theorists in the profession.

\footnotetext{
${ }^{11}$ Op. cit., Jarvie, p. 18.
} 